\title{
PANORAMA IMAGE SETS FOR TERRESTRIAL PHOTOGRAMMETRIC SURVEYS
}

\author{
L. Piermattei ${ }^{a}$, W. Karel ${ }^{b}$, A. Vettore ${ }^{c}$, N. Pfeifer ${ }^{b}$ \\ ${ }^{a}$ University of Padova, Dept. of Land, Environment, Agriculture and Forestry, Italy - livia.piermattei@studenti.unipd.it \\ ${ }^{b}$ Dept. of Geodesy and Geoinformation, TU Wien, Austria - (wilfried.karel, norbert.pfeifer)@geo.tuwien.ac.at \\ ${ }^{c}$ University of Padova, Interdepartment Research Center of Geomatics, Italy - antonio.vettore@unipd.it
}

Commission V, WG V/5

KEY WORDS: Terrestrial photogrammetry, Accuracy, Bundle block adjustment, Panorama images, Georeferencing

\begin{abstract}
:
High resolution 3D models produced from photographs acquired with consumer-grade cameras are becoming increasingly common in the fields of geosciences. However, the quality of an image-based 3D model depends on the planning of the photogrammetric surveys. This means that the geometric configuration of the multi-view camera network and the control data have to be designed in accordance with the required accuracy, resolution and completeness. From a practical application point of view, a proper planning (of both photos and control data) of the photogrammetric survey especially for terrestrial acquisition, is not always ensured due to limited accessibility of the target object and the presence of occlusions. To solve these problems, we propose a different image acquisition strategy and we test different geo-referencing scenarios to deal with the practical issues of a terrestrial photogrammetric survey. The proposed photogrammetric survey procedure is based on the acquisition of a sequence of images in panorama mode by rotating the camera on a standard tripod. The offset of the pivot point from the projection center prevents the stitching of these images into a panorama. We demonstrate how to still take advantage of this capturing mode. The geo-referencing investigation consists of testing the use of directly observed coordinates of the camera positions, different ground control point (GCP) configurations, and GCPs with different accuracies, i.e. artificial targets vs. natural features. Images of the test field in a low-slope hill were acquired from the ground using an SLR camera. To validate the photogrammetric results a terrestrial laser scanner survey is used as benchmark.
\end{abstract}

\section{INTRODUCTION}

The recent evolution of photogrammetry, incorporating incremental bundle adjustment, SIFT keypoints, and dense image matching allows obtaining automatically three-dimensional (3D) information of the photographed object from a sequence of overlapping images taken with a consumer-grade camera. It does neither require a priori knowledge of the interior orientation, nor of the camera positions. This approach is lower in instrument costs compared to light detection and ranging technology (LiDAR), and has lower requirements on user expertise to create a geometrical 3D model thanks to the high level of automation in image processing. Additionally, in different fields of the geosciences (James and Robson, 2014; Thoeni et al., 2014; Piermattei et al., 2015; Smith and Vericat, 2015; Stumpf et al., 2015) it was shown that accuracy and resolution obtained are comparable to those obtained by LiDAR. However, as reported in the investigations on error sources and obtained precision by (Wackrow and Chandler, 2011; James and Robson, 2012; 2014; Micheletti et al., 2014; Nocerino et al., 2014; Bemis et al., 2014) the quality of the photogrammetric results depends principally upon (i) the camera network geometry and (ii) control data. Network geometry comprises the number of photos, the percentage of overlap, how convergent the views are, and the intersection angle. Control data concerns whether ground control points (GCPs), scale measurements, or camera positions are used for datum definition. James and Robson (2012) demonstrated that a poor quality and wrong distribution of the control data can lead to a model distortion, but setting such points at locations well suited for the survey can be difficult or costly (Forlani et al., 2013). Moreover, the design of the imaging network in accordance with the required accuracy, resolution and completeness is still a challenging task in photogrammetry (Alsadik et al., 2013; Nocerino et al., 2013). As a further matter, a weak photogrammetric block design reduces the ability of automated feature matching to identify reliably corresponding features in overlapping images affecting the final accuracy and completeness of the 3D model (Wenzel et al., 2013). In addition, different accuracies can be obtained according to the platform used to capture the images (both terrestrial and aerial), the image quality (resolution, sharpness and light conditions during photo acquisition), and the nature and complexity of the study object. Thus, special care should be taken while planning the photogrammetric survey to optimize the 3D model quality and spatial coverage. From a practical application point of view, a proper planning (of both photos and control data) of the photogrammetric survey especially for terrestrial acquisition is not always ensured due to the limited accessibility of the target object and the presence of occlusions such as vegetation cover or the presence of rock masses.

To deal with the practical issues of a terrestrial photogrammetric survey, we propose a different image acquisition strategy and we extend the previous studies on the georeferencing procedure.

The proposed photogrammetric survey is based on the acquisition of a sequence of images in panorama mode. This means that at each established position a series of pictures with overlapping fields of view are taken by rotating the camera on a standard tripod, to cover the object of interest. Because there is an offset of the pivot point from the projection center, these images cannot be stitched into a panorama. The advantages of this mode of image acquisition were already highlighted by Wenzel et al. (2013) who proposed a practical guideline for acquisition planning but without providing an evaluation of the precision obtainable from a set of images acquired in panorama mode. We tested this strategy to survey natural terrain.

The geo-referencing investigation consists of testing the use of directly observed camera positions with GPS (named GPS-PRCs, i.e. GPS project centers), different GCP configurations, and GCPs with different accuracy, i.e. artificial targets vs. natural features. Images of a test field in a low-slope hill were acquired 
from the ground using an SLR camera. To validate the photogrammetric results a modern terrestrial laser scanner (TLS) survey was used as benchmark and a series of artificial targets measured with a total station were selected as check points (CPs). The commercial software PhotoScan was employed to process the image datasets. OrientAL (Karel et al., 2013), a software developed by TU Wien, was used additionally. Henceforth, we refer to the PhotoScan and OrientAL software using the acronyms of PS and OA, respectively.

\section{METHODS}

To test our image acquisition strategy and to deal with the practical issues of a terrestrial photogrammetric survey we selected a test area located at Torrente Val Montina, Perarolo di Cadore (Italy). The surveyed area is a hill next to the hydroelectric power station which was built at the terminal flat area of the Montina stream, a small branch of the Piave river. An access road to the building adjacent to a wall crosses the area. The investigated area alongside the stream has an extension of 150 meters and covers an area of about $7,300 \mathrm{~m}^{2}$ with an average slope of about $38^{\circ}$. The surface of the hill is characterized at the northern part, close to water, by gravel river debris of different sizes and at the southern part by shrub cover. The investigations were carried out over the entire reconstructed area and on two sub-areas with and without vegetation. The test survey took place on August 4, 2014. The following surveying methodology was adopted to acquire the dataset: i) topographic survey with total station to measure the control data; ii) static positioning GNSS survey to perform the datum transformation; iii) terrestrial laser scanning acquisition of the area of interest and the artificial targets; iv) terrestrial photogrammetric survey acquiring the images in panorama mode and single photos; v) GPS-PRCs in real time kinematic (RTK). Data acquisition and photogrammetric workflow to produce the dense point clouds are described in the next section.

\subsection{Topographic and TLS data acquisition and processing}

The data acquisition started with the planning of target positions. We used 17 black/white targets mounted on a vertical planar support and two tripod-mounted Leica targets (i.e. 6" circular blue/white target). The Black and white targets, used for photogrammetric purposes, were distributed within the area of interest. Due to accessibility and safety issues it was impossible to achieve a proper placing of these targets for the photogrammetric survey (i.e. covering the 3D extent of the area). A first line of 12 targets was distributed along the road and a second parallel line of 5 targets the north-west part of the hill, close to the river. The two circular targets were surveyed by dGPS measurements using a dual frequency GPS/GLONASS receiver (Topcon Hiper Pro) to geo-reference the data in the global coordinate system UTM-WGS84, zone 33N.

The targets (both black/white and circular) were measured by means of a total station and of the TLS. The total station measurements were carried out with a reflectorless Leica TPS700 from one position, considering a maximum distance to the black and white targets of $95 \mathrm{~m}$. The total station coordinates of the black and white targets were used for the image processing. Eight of these targets were used as GCPs in the bundle adjustment, and the other points as CPs (Figure 1). RMSE of the target centers measured by total station compared to those surveyed by TLS was $0.013 \mathrm{~m}$. A ScanStation C10 TLS was employed to generate a reference point cloud to validate the photogrammetric survey.

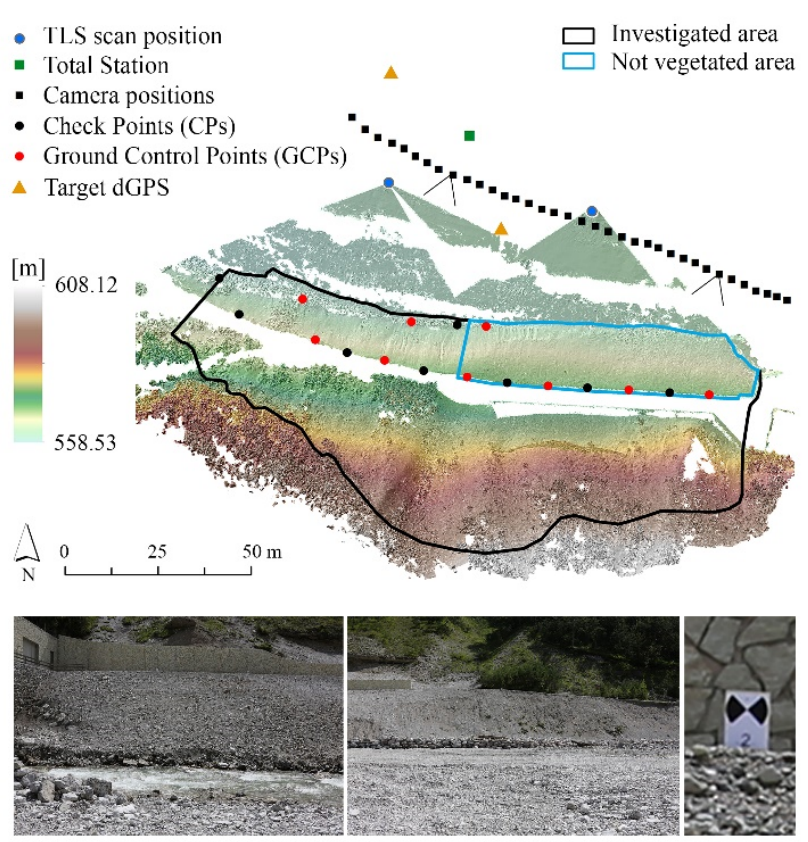

Figure 1. Top: configuration of topographic surveys and TLS survey, and camera network geometry. TLS elevation model with the GCPs (red points) and CPs (black points). The black

line represents the investigated area and the blue line the perimeter of area not covered by vegetation. Bottom: images of the test area and a black and white flat target for the photogrammetric survey.

TLS measurements were performed from two scan positions, located at mean distance of $50 \mathrm{~m}$ from the object. Target-based registration using both black and white flat targets and circular targets provided a first registration error of $0.019 \mathrm{~m}$. The ICP optimization implemented in Leica Cyclone 8.0 produced an RMS value of $0.012 \mathrm{~m}$.

The GPS-PRCs were measured by the following combination. As the images were taken from a tripod, the tripod plumb points were measured by GPS in Real Time Kinematic (RTK). The height of the camera tripod head (measured with a handheld laser range finder) was added to determine the coordinates of the projection centers.

\subsection{Photogrammetric workflow}

2.2.1 Data acquisition: Overlapping images were taken with a Canon EOS5D Mark III (28 mm focal length, sensor size of $36.0 \times 24.0 \mathrm{~mm}$ ) attached to a tripod to avoid camera shake and to ensure a common pivot point for panorama image sets. The acquisition mode of the images was twofold. From each individual camera position, a first normal image of the investigated surface was taken, keeping the direction of the camera's optical axis almost perpendicular to the surface. Then, to cover the whole surface from these positions, additional images were taken in panoramic mode, which involved taking a series of overlapping photographs by rotating the camera on the tripod from left to right.

The sequence of images was acquired maintaining an average depth distance of about $50 \mathrm{~m}$ from the object, yielding a mean GSD of $0.02 \mathrm{~m}$. It was composed by 36 consecutive camera positions that implied 376 images for the panoramas from these positions (around 7 to 14 photos per position). The mean baseline between adjacent camera positions was about $3 \mathrm{~m}$. 

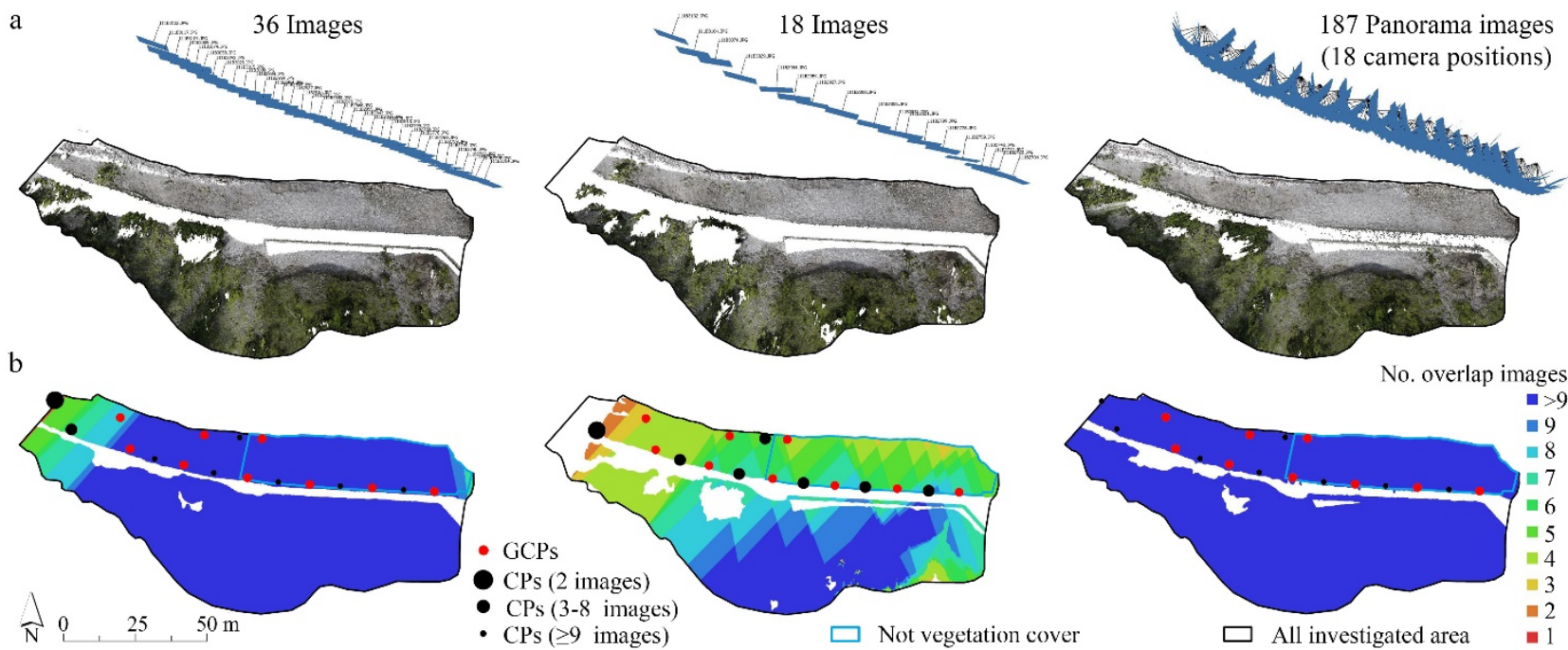

Figure 2: Acquisition geometry for the experimental tests. a) Dense point cloud generated with PS and b) image coverage of the photogrammetric survey for each camera configuration: single (left, center) and panorama images (right). The black points represent the check point (CPs) and their dimension is related to the number of images that view that point.

2.2.2 Data processing: To test the panorama image acquisition, a subset of the entire dataset was selected to reduce the amount of panorama images. Therefore, 18 camera positions of the entire sequence ( 36 images) were chosen including the first and the last station. Consequently, the mean baseline between adjacent images was 8 meters. The normal images acquired from each of these positions were included in the panorama dataset during the processing, resulting in 187 images in total (Figure 2a, right).

The dataset of 36 images was processed separately eight times testing i) GPS-PRCs, ii) natural features employed as GCPs whose coordinates were extracted from the TLS point cloud, and iii) different GCP configurations i.e. number and distribution of artificial targets measured with topographic instrumentation.

In this work the commercial software PhotoScan Pro (v. 1.1.5) was used to process all the datasets (both panorama images and normal images). PS applies the structure from motion (SfM) technique which produces the internal camera parameters (inner orientation), the relative orientation of cameras at the time of image acquisition, and a 3D sparse point cloud of typically a few thousand points, which represents the valid match points between the photographs and the structure of the scene (De Reu et al., 2013). The key components of the photo-based reconstruction workflow with PS are (i) GCP identification in the images (ii) SfM step, and (iii) dense point cloud reconstruction with the Multi-View Stereo (MVS) algorithm. While the software is highly automated, it offers few means of user-control, which are limited to some pre-processing steps like the manual masking of moving objects (e.g. water and clouds) and to the bundle adjustment optimization after the initial camera orientation. In addition, camera positions and camera calibration parameters can be included during the camera orientation. These latter constraints were tested beforehand to identify the better processing of the panorama images with PS, which was based on a 'self-calibration' of the cameras by including the GCPs in the bundle adjustment optimization, without any others constraints. Furthermore, the masking of the foreground in the panorama images improved the camera orientations. The dense image matching (of all experimental datasets) was done by processing the images at half resolution.

In addition to PS, the orientation and camera calibration of the 18-image dataset and the panorama images were estimated using OrientAL, a research-based software package developed at TU
Wien, followed by dense image matching using SURE (Rothermel et al., 2012). With OA, the prepared image masks were not needed, and the camera was calibrated on-the-job, separately for each image data set. The processing by OA started with a variation of SfM that uses affine invariant feature points and introduces a variable subset of distortion parameters, as feasible. Having applied the appropriate similarity transform to the result of incremental reconstruction, GCP image observations were introduced with a higher weight than for feature points due to their better definition quality, and GCP object coordinates were introduced as stochastic variables. During the capturing of panorama images, the camera was only rotated about the vertical axis of the tripod, while leaving the other rotation axes of the tripod head fixed. This results in the offset vector between the projection centre and the pivot point being constant in the camera coordinate systems of panorama images. Additionally, the angle between the offset vector and the axis of rotation results as constant. The position of the pivot point and the direction of the rotation axis in object space change every time the tripod is relocated, but the offset vector in the camera coordinate system and its angle with the rotation axis remain unchanged. While the incorporation of this knowledge into the bundle block adjustment increased the number of unknowns by 5 for each tripod position, and additionally by 3 for the whole block, modelling the relative orientations of panorama images turned out to significantly enhance the quality of results.

\subsection{Methods of accuracy assessment}

Accuracy assessment of the photogrammetric results was performed considering both point cloud and digital elevation model (DEM). Even though both photogrammetric packages provide DEM reconstruction, the same interpolation method was adopted to create the elevation model of the TLS and the photogrammetric point cloud. These were created using Natural Neighbours interpolation with a pixel size of $0.05 \times 0.05 \mathrm{~m}$. For comparison purposes, areas without information were masked and not interpolated while converting point clouds into DEMs. The photogrammetric and the TLS 3D models were cropped to include only the area of interest. Furthermore, the analyses were performed for the entire investigated area and for a selected area without vegetation cover (Figure 2b). We assumed as reference 
data the TLS dataset and the 8 CPs. Considering the dataset the following accuracy analyses were performed:

- Root Mean Square Error (RMSE) of the computed object coordinates with respect to $\mathrm{CP}$ coordinates. We used the reconstructed, geo-referenced camera orientations to forward intersect the image observations of the CPs. Those forward intersected points were compared to the ones measured with the total station. The RMSE was evaluated for all CPs and in relation to the number of images (intersecting optical rays) that view these points (i.e. 2 images, 3-8 images and $>9$ images) (Figure 2b).

- Absolute distance (cloud to cloud distance, C2C) between the TLS point cloud and the photogrammetric point cloud comparing both the sparse point cloud (tie points) and the dense point cloud. - DEM of difference (DoD) between the TLS and the photogrammetric DEMs in the overlapping areas.

The main statistics as mean, standard deviation $(\sigma)$ and RMSE for $\mathrm{C} 2 \mathrm{C}$ and $\mathrm{DoD}$ were derived. The spatial distribution of the error was also taken into account to describe the accuracy of the final DEMs.

\section{RESULTS}

\subsection{Accuracy assessment regarding image acquisition}

The photogrammetric dataset and the main results of the image processing based on the camera acquisition setup are shown in tables 1 and 2. A first accuracy analysis was to compare the sparse point clouds with the laser scanning point cloud in terms of absolute distances ( $\mathrm{C} 2 \mathrm{C}$ in Table 1). The elevation of the panorama images sparse point cloud is on average higher than the TLS point cloud by $2.7 \mathrm{~cm}$, with a variation of $\pm 5.8 \mathrm{~cm}$. The 36 images orientation produced similar results in terms of mean, 2.5 $\mathrm{cm}$, and standard deviation, $4.0 \mathrm{~cm}$, with a comparable number of tie points. The 18 single images configuration provided a slightly lower accuracy $(3.2 \mathrm{~cm} \pm 6.2 \mathrm{~cm})$ with also a lower number of extracted tie points. The accuracy estimation of the photogrammetric 3D model after dense point cloud reconstruction was performed by analysing the error statistics of both DoD and C2C comparison. The accuracies for the whole investigated area obtained by processing either the panorama images or the 18 single images, are practically identical to those obtained by processing 36 images (Table 2). In the area not covered by vegetation, the 18 single images acquisition produced a higher $\sigma$ and RMSE compared to the other two datasets. The dispersion around zero is lower in the panorama imagery in comparison to the single views acquired from the same positions: $86 \%$ of the height differences fall in the range $\pm 0.05 \mathrm{~m}$ for the 18 images dataset, whereas the percentage increases to about $98 \%$ for both the panorama images and the 36 images. Furthermore, the 3D model generated from 18 single images was incomplete as visible in the spatial distribution of elevation differences (Fig. 3, "18 Images"). The map of the z-differences suggests a deformation (bending effect) in the $3 \mathrm{D}$ results obtained by processing the single images, both from 18 and 36 camera positions (Figs. 3a and 3b). This deformation, strongest at the borders of the $3 \mathrm{D}$ model is also demonstrated by the high error $(0.18 \mathrm{~m})$ of the check point located in that area. The OrientALbased results are rather similar to those obtained by PS. The statistics analysis on the sparse point cloud was performed considering the tie-points observed in at least three photographs. The bundle adjustment estimated the standard deviations for each point of the sparse point cloud. The plotting of the median of the standard deviations for the Z-coordinates of all points for the 18images and the panorama images is shown in Figure 3c. No significant differences are visible in the standard deviation distributions of the two datasets. The dense point cloud was subsampled at $2 \mathrm{~cm}$ to reduce the number of points before the comparison. The accuracy assessment of the panorama images reconstruction shows a slightly lower quality for the entire investigated area. However, the distribution of the z-difference for the panorama images-dataset reveals less distortion also in the vegetation-free coverage. The 18 images orientation with $\mathrm{OA}$ provided higher accuracy in comparison with PS (Tables 1 and 2, and Fig. 3a).

\begin{tabular}{|c|c|c|c|c|c|c|c|c|c|c|c|c|}
\hline \multicolumn{13}{|c|}{ SfM results } \\
\hline \multirow{2}{*}{$\begin{array}{l}\text { Camera } \\
\text { acquisition }\end{array}$} & \multicolumn{2}{|c|}{$\begin{array}{l}\text { Reprojection } \\
\text { error [pixel] }\end{array}$} & \multirow{2}{*}{$\begin{array}{l}\text { No. of tie } \\
\text { points } \\
\text { [pts] }\end{array}$} & \multicolumn{2}{|c|}{$\begin{array}{l}9 \text { GCPs } \\
\text { RMSE }\end{array}$} & \multicolumn{4}{|c|}{8 CPs RMSE [m] } & \multicolumn{3}{|c|}{$\begin{array}{c}\mathrm{C} 2 \mathrm{C} \\
\text { sparse point cloud }[\mathrm{m}]\end{array}$} \\
\hline & Mean & Max & & {$[\mathrm{m}]$} & [pixel] & All images & 2 images & 3-8 images & $\geq 9$ images & Mean & $\sigma$ & RMSE \\
\hline \multicolumn{13}{|c|}{ PhotoScan } \\
\hline 187 panorama & 0.407 & 1.222 & 115,055 & 0.032 & 0.522 & & - & - & 0.029 & 0.027 & 0.058 & 0.064 \\
\hline 18 images & 0.357 & 1.100 & & 0.030 & 0.261 & & 0.065 & 0.026 & - & 0.032 & 0.062 & 0.070 \\
\hline 36 images & 0.390 & 1.049 & 119,719 & 0.015 & 0.321 & 0.068 & 0.183 & 0.034 & 0.019 & 0.025 & 0.040 & 0.047 \\
\hline \multicolumn{13}{|c|}{ OrientAL } \\
\hline 187 panorama & 0.394 & 1.401 & 68,345 & 0.001 & 0.673 & 0.019 & - & - & 0.019 & 0.028 & 0.072 & 0.077 \\
\hline 18 images & 0.201 & 1.064 & 18,100 & 0.001 & 0.251 & 0.157 & 0.413 & 0.013 & - & 0.027 & 0.083 & 0.087 \\
\hline
\end{tabular}

Table 1. Dataset characteristics and image processing results for single images acquisition vs. panorama acquisition using PS and OA.

\begin{tabular}{|c|c|c|c|c|c|c|c|c|c|c|c|c|}
\hline \multirow{3}{*}{$\begin{array}{l}\text { Camera } \\
\text { acquisition }\end{array}$} & \multicolumn{4}{|c|}{ DoD $[\mathrm{m}]$} & \multicolumn{2}{|c|}{ No. of dense point cloud [pts] } & \multicolumn{6}{|c|}{ C2C dense point cloud [m] } \\
\hline & \multicolumn{2}{|c|}{$\begin{array}{c}\text { All investigated } \\
\text { area }\end{array}$} & \multicolumn{2}{|c|}{$\begin{array}{c}\text { Not vegetated } \\
\text { area }\end{array}$} & \multirow{2}{*}{$\begin{array}{c}\text { All investigated } \\
\text { area }\end{array}$} & \multirow{2}{*}{$\begin{array}{c}\text { Not vegetated } \\
\text { area }\end{array}$} & \multicolumn{3}{|c|}{$\begin{array}{c}\text { All investigated } \\
\text { area }\end{array}$} & \multicolumn{3}{|c|}{$\begin{array}{c}\text { Not vegetated } \\
\text { area }\end{array}$} \\
\hline & Mean & $\sigma$ & Mean & $\sigma$ & & & Mean & $\sigma$ & RMSE & Mean & $\sigma$ & RMSE \\
\hline \multicolumn{13}{|c|}{ PhotoScan } \\
\hline 187 panorama & -0.031 & 0.504 & 0.009 & 0.023 & $21,587,050$ & 4,340 & 0.048 & 0.104 & 0.114 & 0.016 & 0.015 & 0.022 \\
\hline & -0.04 & 0.4 & 0.003 & 0.0 & & & 0.042 & 0.092 & 0.1 & 0.023 & 0.017 & 0.028 \\
\hline 36 images & -0.033 & 0.507 & 0.002 & 0.022 & $19,296,390$ & $4,808,269$ & 0.040 & 0.086 & 0.095 & 0.014 & 0.013 & 0.019 \\
\hline \multicolumn{13}{|c|}{ OrientAL } \\
\hline $187 \mathrm{p}$ & 0.066 & 0.543 & 0.006 & 0.045 & $10,683,701$ & & 0.064 & 0.218 & 0.227 & 0.014 & 0.052 & 0.054 \\
\hline 18 images & 0.012 & 0.479 & 0.016 & 0.022 & $7,695,620$ & $2,375,200$ & 0.038 & 0.092 & 0.099 & 0.015 & 0.011 & 0.019 \\
\hline
\end{tabular}

Table 2. Accuracy assessment based on the elevation difference (DoD) and the absolute distance (C2C) between the photogrammetric data and the TLS reference data for each camera acquisition dataset processed by PS and OA. The TLS acquisition provided $21,292,050$ and 6,235,258 points for the entire investigated area and for the area not covered by vegetation, respectively. 


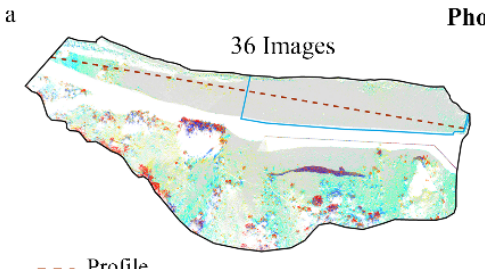

- - Profile

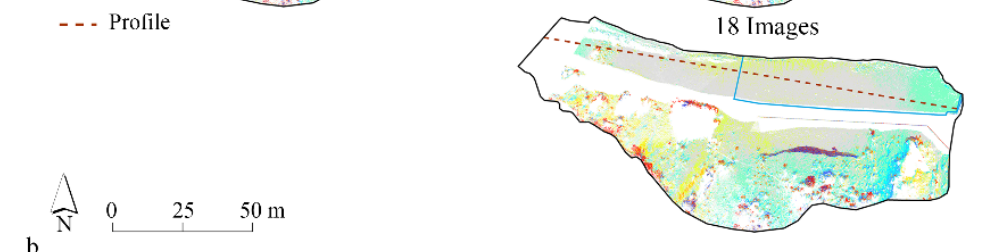

$\mathrm{b}$

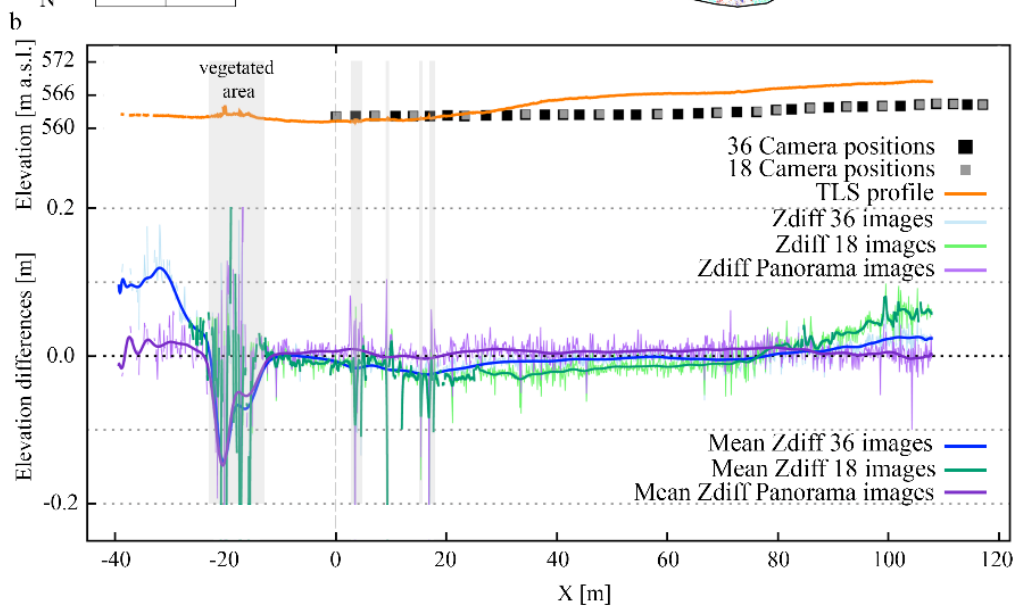

187 Panorama images

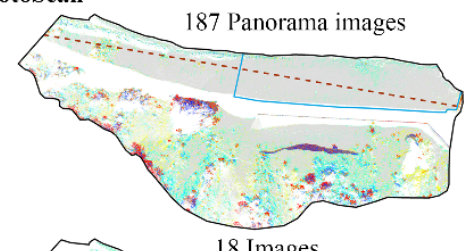

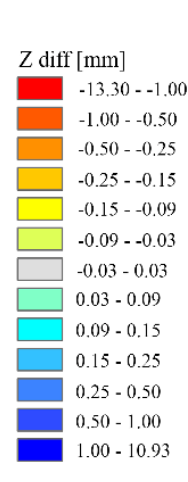
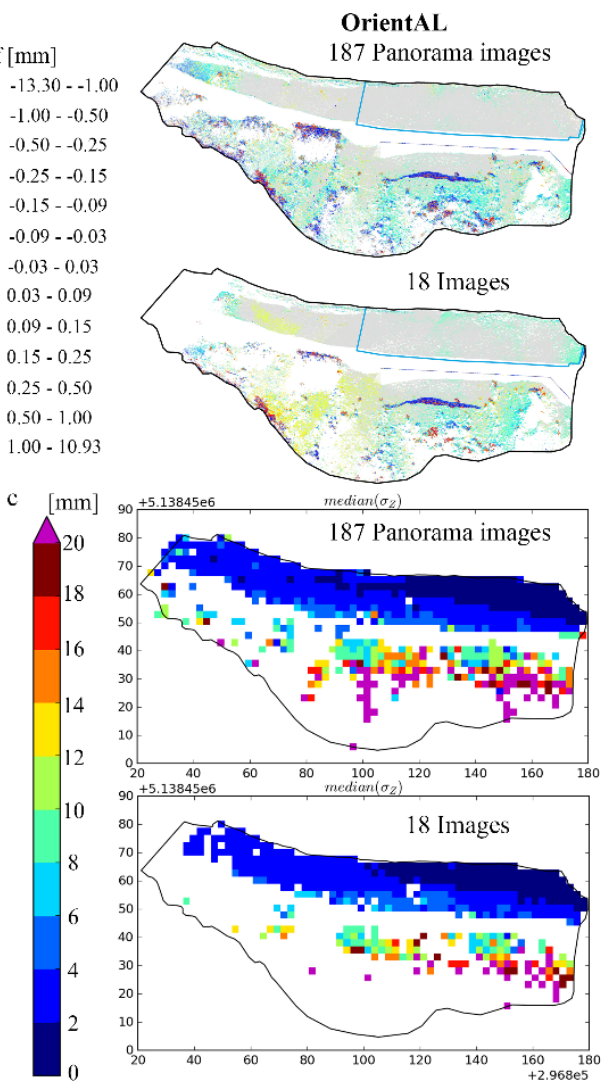

Figure 3. a) DoD between TLS data and the DEM derived from each image dataset processed using PS and OA. b) Profiles show the elevation and camera positions (top) and the elevation difference between PS and TLS-based DEMs (bottom). The location of the profile (red dashed line) is indicated in Fig. 3a. The origin of the $\mathrm{x}$-axis is at the first camera position (in the West) and the minimum and maximum values of the z-differences are set to $-0.2 \mathrm{~m}$ and $0.2 \mathrm{~m}$, respectively. The mean of elevation differences are represented as a smoothed lines with more saturated colors. c) Colored maps of the median of the standard deviations of Z-coordinates of all tie points estimated by OA during the bundle adjustment for the panorama images and the 18-images.

\subsection{Accuracy assessment regarding to the geo-referencing approach}

To test the influence of the geo-referencing approach on the image-based reconstruction the 36 images data set was processed. Geo-referencing based on the 9 artificial targets, being the GCPs already used above, is named in this section 'Configuration A' and we use respective results as reference for the others tests. SfM results for the different experimental setups are shown in Table 3. A first investigation was performed on the feasibility of using the GPS-PRCs of the cameras to scale and geo-reference the image block. Since no reference data are available for the camera positions, the camera coordinates measured with RTK-GPS were compared with the camera positions estimated by PS after the bundle block adjustment.

The largest discrepancies between the GPS-PRC measurements and the projection centers from PS were found for the $\mathrm{y}$ coordinate (depth value) of the cameras with a mean offset of $0.068 \mathrm{~m}$, in comparison to $-0.006 \mathrm{~m}$ and $0.035 \mathrm{~m}$ for the $\mathrm{x}$ and $\mathrm{z}$ coordinates, respectively (total RMSE is $0.093 \mathrm{~m}$ ). The inaccuracy of the GPS-PRCs produced a shift and rotation of the final 3D model as visible in the spatial distribution of the elevation difference between SfM and TLS based DEM (Fig. 4, "GPS-PRCs"). To solve this shift error, we used the TLS point cloud to co-register the photogrammetric point cloud using the ICP algorithm and to extract the coordinates of some natural features employed as GCPs for combining direct geo-referencing with GCPs method. A version of the ICP algorithm tailored to topographic point clouds (Glira et al., 2015) was applied. The co- registration with the TLS point cloud minimized the orientation error of the photogrammetric model. Furthermore, even better results were achieved in terms of error statistics and spatial distribution of the elevation differences than those obtained from processing the images with GCPs (Table 4 and Fig. 4, "GPSPRCs after ICP", "GCPs Configuration A"). Incorporating into the bundle adjustment the GPS-PRCs and 4 natural GCPs located in the vegetation-free area reduced the tilt problem, but some distortions in the 3D model are visible in comparison with the reference TLS DEM (Fig. 4, "GPS-PRCs + 4 Natural Features"). To evaluate the influence of manual identification of natural GCPs, 9 points in the TLS point cloud and in the images were selected. These points were identified close to the artificial targets (Configuration A) in order to maintain the same control data distribution. The transformation residual error of the GCPs identification range from $0.019 \mathrm{~m}$ to $0.086 \mathrm{~m}$ (RMSE error was $0.046 \mathrm{~m}$ and 1.00 in pixel). Despite this error, no significantly lower accuracies were found in comparison to the results obtained by using artificial targets. To quantify the effect of ground control point location, five different GCP configurations (named A, B, C, D, E), changing the number and the distribution of the GCPs, were compared (Fig. 4). Analysis of the GCP residuals suggested that the georeferencing had a RMSE of less than $2 \mathrm{~cm}$ for all GCP configurations. However, these statistics can be used as a first indication of accuracy of the network configuration, because these points were used to compute the solution of the network (Wachrow and Chandler, 2011) and therefore, the residual values show how well the data fit to the ground control points (Dietrich, 2015). In this work, the accuracy 
of the point cloud evaluated at the CPs is highly affected by the number of images that observe the area where the check point is located rather than by GCP distribution. The map of z-error demonstrated strong model distortions in the configurations B, D and $\mathrm{E}$ caused by inappropriate GCPs distribution (i.e. aligned or clustered, Fig. 6). These distortions are not highlighted in the accuracy estimation based on the mean and $\sigma$ of DoD, with the exception of the mean value of configuration $B$ with $-0.16 \mathrm{~m}$ calculated for the entire area (Table 4). For this test, a more reliable evaluation of the $3 \mathrm{D}$ model accuracy is provided by the absolute distance analysis between the photogrammetric point cloud and the reference TLS point cloud (C2C in Table 4). Configuration $\mathrm{C}$ characterized by four GCPs located on the boundary of the investigated area provided identical results of using 9 GCPs (Configuration A) but with a lower dispersion of elevation differences around zero, with $65 \%$ of values that range between $+/-0.03 \mathrm{~m}$ in comparison to $63 \%$ obtained for the configuration A.

\section{SfM (PS) results}

\begin{tabular}{|c|c|c|c|c|c|c|c|c|c|}
\hline \multicolumn{10}{|c|}{$\begin{array}{r}\text { SIIV (PS) results } \\
\end{array}$} \\
\hline \multirow[t]{2}{*}{ Georeferencing method } & \multirow{2}{*}{$\begin{array}{l}\text { No. } \\
\text { GCPs }\end{array}$} & \multicolumn{2}{|c|}{$\begin{array}{c}\text { Reprojection error } \\
\text { [pixel] }\end{array}$} & \multicolumn{2}{|c|}{9 GCPs RMSE } & \multicolumn{4}{|c|}{8 CPs RMSE [m] } \\
\hline & & Mean & Max & {$[\mathrm{m}]$} & [pixel] & All images & 2 images & 4 images & $>9$ images \\
\hline GPS-PRCs & - & 0.389 & 1.055 & - & - & 0.104 & 0.227 & 0.119 & 0.059 \\
\hline GPS-PRCs + Natural GCPs & 4 & 0.392 & 1.042 & 0.039 & 0.461 & 0.063 & 0.086 & 0.058 & 0.059 \\
\hline Natural GCPs & 9 & 0.392 & 1.047 & 0.046 & 1.000 & 0.058 & 0.134 & 0.027 & 0.038 \\
\hline A & 9 & 0.390 & 1.049 & 0.015 & 0.321 & 0.068 & 0.183 & 0.034 & 0.019 \\
\hline $\mathrm{B}$ & 3 & 0.390 & 1.068 & 0.003 & 0.288 & 0.082 & 0.161 & 0.032 & 0.067 \\
\hline $\mathrm{C}$ & 4 & 0.391 & 1.061 & 0.016 & 0.295 & 0.063 & 0.169 & 0.019 & 0.021 \\
\hline D & 6 & 0.390 & 1.086 & 0.009 & 0.342 & 0.064 & 0.162 & 0.051 & 0.024 \\
\hline$\underline{E}$ & 3 & 0.392 & 1.073 & 0.010 & 0.237 & 0.053 & 0.110 & 0.023 & 0.054 \\
\hline
\end{tabular}

Table 3. Dataset characteristics and image processing results for the tested geo-referencing approaches.

\begin{tabular}{|c|c|c|c|c|c|c|c|c|c|c|c|}
\hline \multirow{3}{*}{ Georeferencing method } & \multirow{3}{*}{$\begin{array}{c}\text { No. } \\
\text { GCPs }\end{array}$} & \multicolumn{4}{|c|}{ DoD $[\mathrm{m}]$} & \multicolumn{6}{|c|}{$\mathbf{C 2 C}[\mathrm{m}]$} \\
\hline & & \multicolumn{2}{|c|}{$\begin{array}{c}\text { All investigated } \\
\text { area }\end{array}$} & \multicolumn{2}{|c|}{$\begin{array}{c}\text { Not vegetated } \\
\text { area }\end{array}$} & \multicolumn{3}{|c|}{$\begin{array}{l}\text { All investigated } \\
\text { area }\end{array}$} & \multicolumn{3}{|c|}{$\begin{array}{c}\text { Not vegetated } \\
\text { area }\end{array}$} \\
\hline & & Mean & $\sigma$ & Mean & $\sigma$ & Mean & $\sigma$ & RMSE & Mean & $\sigma$ & RMSE \\
\hline GPS-PRCs & - & 0.036 & 0.506 & 0.037 & 0.026 & 0.066 & 0.087 & 0.109 & 0.029 & 0.015 & 0.033 \\
\hline GPS-PRCs * & - & -0.042 & 0.506 & 0.000 & 0.024 & 0.039 & 0.086 & 0.095 & 0.015 & 0.013 & 0.020 \\
\hline GPS-PRCs + Natural GCPs & 4 & -0.024 & 0.507 & 0.003 & 0.026 & 0.046 & 0.086 & 0.098 & 0.016 & 0.014 & 0.021 \\
\hline Natural GCPs & 9 & -0.041 & 0.508 & 0.007 & 0.026 & 0.040 & 0.086 & 0.095 & 0.016 & 0.014 & 0.022 \\
\hline A & 9 & -0.033 & 0.507 & 0.002 & 0.022 & 0.040 & 0.086 & 0.095 & 0.014 & 0.013 & 0.019 \\
\hline B & 3 & -0.163 & 0.539 & 0.002 & 0.045 & 0.094 & 0.100 & 0.137 & 0.028 & 0.019 & 0.034 \\
\hline $\mathrm{C}$ & 4 & -0.038 & 0.504 & -0.005 & 0.022 & 0.040 & 0.086 & 0.095 & 0.015 & 0.013 & 0.019 \\
\hline D & 6 & 0.006 & 0.501 & -0.030 & 0.029 & 0.062 & 0.089 & 0.109 & 0.027 & 0.017 & 0.033 \\
\hline E & 3 & 0.008 & 0.507 & 0.043 & 0.032 & 0.055 & 0.086 & 0.102 & 0.034 & 0.021 & 0.039 \\
\hline
\end{tabular}

Table 4. Accuracy assessment based on the elevation difference (DoD) and the absolute distance $(\mathrm{C} 2 \mathrm{C})$ between the photogrammetric data and the TLS reference data for each geo-referencing experiment. * After ICP registration with the TLS point cloud.

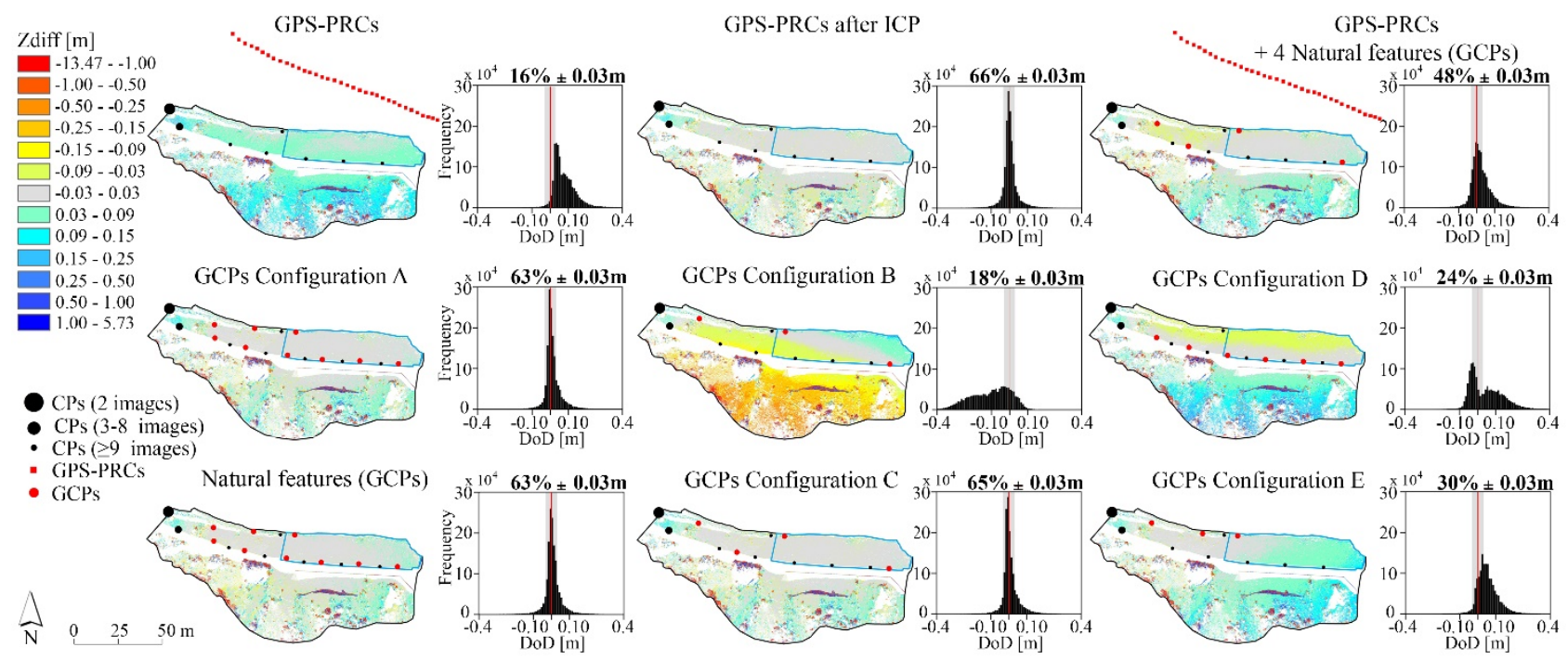

Figure 4. The spatial distribution of the elevation differences (DoD) between TLS data and each photogrammetric DEM obtained by processing the images with different georeferencing methods. The frequency distribution histograms extracted from the DoDs are provided for the entire investigated area. 


\section{DISCUSSION}

The results of Section 3.1 show the feasibility of using images acquired in panorama mode to reconstruct the surface topography with the SfM approach. The proposed strategy improved the quality of the SfM results in comparison with those obtained from the single images dataset acquired from the same position and from the two-fold positions. From a practical point of view, this method allows to acquire the images in an easy way without a proper planning of the overlap between consecutive cameras. Furthermore it allows to reduce the number of camera stations necessary to reconstruct the entire area. Moreover, the panorama images acquisition required little additional time in comparison to the single images acquisition, and no specialized panoramic camera head.

The advantages of the panorama acquisition in the SfM reconstruction are i) the high redundancy of the observations (i.e. how often a surface point is seen), ii) the increase of the baseline between images that view the same points that implies an increase of the intersection angles of optical rays, iii) the presence of multiple convergent images in the imaging geometry, and iv) less risk to have incomplete spatial coverage. These characteristics increase the quality of the 3D model reconstruction, as recently reported in literature. Indeed, as shown in (Rumpler et al., 2014) the mean accuracy increases with a higher number of image measurements, although there is a saturation on accuracy improvement within larger datasets. Similarly, Wenzel et al. (2013) demonstrated an improvement of the precision with increasing the intersection angles and redundancy. Camera configurations with large intersection angles enhance the depth precision, but reduce the image similarity causing lower matching performance and consequently lower point density, outliers or incompleteness of the 3D model (Wenzel et al., 2013). Therefore, increasing the number of observations by rotating the camera at the same position leads to high completeness. Additionally, the importance and use of convergent image networks is demonstrated by (Remondino and Fraser, 2006, Wackrow and Chandler, 2011; James and Robson, 2014; Bemis et al.; Nocerino et al., 2014). Convergent imagery reduces the systematic error caused by inaccurate lens distortion parameters. Conversely, parallel geometry allows SfM algorithm to accumulate error from radial distortion (Dietrich, 2015) and in case of open sequences of photographs acquired in a single strip, the bending effect can appear (Nocerino et al., 2014).

Both photogrammetric software provided similar results with higher quality of the $3 \mathrm{D}$ model of the panorama images in comparison to the single images. The increase of the $3 \mathrm{D}$ model accuracy of the panorama images is defined by the higher number of points with higher accuracy according to TLS reference data, and which are distributed fairly evenly over the entire reconstructed area. Additionally, the elevation differences between the SfM and the TLS DEM show a lower distortion on the border of the reconstructed area in comparison to the model generated from 36 single images. The single views 3D model produced a bending effect on the border that is more evident in the model produced by a smaller number of camera positions. For the 18 single images dataset, the bending effect is higher in the PS model rather than OA, as well as the DoD-mean value $(4 \mathrm{~cm}$ vs $1.2 \mathrm{~cm}$ with PS and OA, respectively). Our results validate that parallel geometry of the images causes deformation and that the error is higher where the number of overlapping images is lower. Moreover, the error increases outside the area covered by the GCPs. The RMSE of the CP located outside this area is greater than $10 \mathrm{~cm}$ for the reconstructed surface from single images. The model distortion could be solved by positioning GCPs there. Low overlap also leads to incomplete coverage of the 3D model, as demonstrated by the DEM reconstructed by OA and PS using 18 single images. The higher redundancy of the panorama acquisition increases the spatial coverage but also the number of tie points and dense point clouds in comparison to the single images acquisition. The oblique views in panorama mode allow reconstructing areas that are occluded by vegetation and therefore not visible in a normal view of the object. The slightly lower number of the dense matching points that we obtain from the 'panorama' model in the vegetation-free area (close to the river) is probably caused by the different image masks of the river water and of the foreground used to process these images.

The necessity to mask in PS the foreground for all panorama images represents a first drawback of this strategy as it is a timeconsuming task. Specifically, in our test the camera orientation without foreground masked (only the water of the river) provided worst results (i.e. not all images were properly oriented). Secondly, the high number of photographs uploaded requires more processing time and high computer performance, in particular for the dense matching computation in PS. Contrary to PS, the panorama images orientation in OA did not suffer from these problems, and no masking of the images was necessary. Moreover, as the rotation of the camera was modelled explicitly, OA's results are more reliable.

For all reconstructed image-based models, the highest values of elevation difference are identified on the vegetated area and on the undercut area where, however, a vertical difference is not appropriate for accuracy evaluation. In SfM approach, vegetation represents a general limitation not only for occlusion but also for degradation in the quality of surface reconstruction (Micheletti et al., 2014; Dietrich, 2015) due to the high surface roughness and variable pattern (Stumpf et al., 2015). In our work, the standard deviation of the elevation differences and distance 'cloud to cloud' with the TLS is significantly higher than the mean values in the vegetated area. However, the mean values close to zero of the DoD analysis are not representative as an accuracy parameter because they are related to the presence of negative and positive deviations that are rather spatially clustered. Therefore, a spatial distribution of elevation differences between photogrammetry and TLS DEMs was considered to be essential for the accuracy analysis. A positive trend of elevation differences is shown in the southern part of the area. This could be caused by the larger distance from the camera positions and by the not optimal distribution of GCPs to estimate the correct rotation of the surface.

The investigations in this paper highlighted a significant influence of the distribution of ground control points used for georeferencing on the SfM results. In particular, and in line with expectations, we demonstrated that a weak GCP distribution, like aligned or clustered GCPs, generated model distortions, providing good accuracy only within the area covered by the GCPs. A higher number of GCPs (Configuration A versus Configuration C) did not improve the photogrammetric results as also demonstrated by (Nocerino et al. 2014) and (James and Robsons 2014). Furthermore, the final SfM accuracy is more affected by the GCP distribution than their accuracy, for example by using some natural features employed as GCPs whose coordinates were manually extracted from the TLS point cloud. As an alternative to GCP-based referencing we measured the GPS camera centers that were employed as control data. The resulting photogrammetric accuracy depends on the accuracy of GPS measurements. However, despite this inaccuracy, the 3D model showed no distortion or systematic error. The linear arrangement of camera positions left one rotation of the datum weakly defined. Therefore, an ICP registration with the reference TLS data was successfully used to compensate the transformation error (both translation and rotation) of the photogrammetric block. This solution provided the highest accuracy compared to other GCP-based geo-referencing tests. 


\section{CONCLUSIONS}

The main contribution of this work is to propose a terrestrial photogrammetric survey based on the acquisition of a sequence of images in panorama mode. This means that at each established step the camera mounted on a tripod is rotated to photograph the entire object. The main conclusion of this study is that the proposed strategy has improved the accuracy, resolution and completeness of the SfM results in comparison with those obtained from a single image acquisition. The increased number of overlapping and convergent images reduced the distortions of the 3D model where GCPs are not located and where a lower number of intersection rays is available. Panorama images provided a complete spatial coverage of the $3 \mathrm{D}$ model while single images from the same positions led to incomplete surface coverage. Furthermore, the panorama acquisition increased the number of viewpoints reducing the data gaps caused by the presence of occlusions such as vegetation cover. From a practical point of view, using panoramic image sequences requires only little additional effort in the field and it allows to reduce the number of camera stations necessary to reconstruct the entire area. The disadvantage of masking the panorama images in PS was solved by OA.

Regarding the geo-referencing, the direct measurement of camera positions using GPS is more convenient than usage of GCPs, if a proper placing of GCPs is not ensured. However, the ICP registration should be applied to solve the transformation error introduced by the direct measurements, if a reference surface is available.

\section{REFERENCES}

Alsadik, B., Remondino, F., Menna, F., Gerke, M., \& Vosselman, G. 2013. Robust extraction of image correspondences exploiting the image scene geometry and approximate camera orientation. ISPRS Int. Arch. Photogramm. Remote Sens. Spat. Inf. Sci, Vol. 40(5/W1), pp. 1-7, doi:10.5194/isprsarchives-XL-5-W1-1-2013.

Bemis, S., Micklethwaite, S., Turner, D., 2014. Ground-based and UAV-Based photogrammetry: a multi-scale, high-resolution mapping tool for Structural Geology and Paleoseismology. J Struct Geol., 69, pp. 163-178, doi:10.1016/j.jsg.2014.10.007.

De Reu J, Plets G, Verhoeven G, De Smedt P, Bats M, Cherretté B, De Clercq W. 2013. Towards a three-dimensional cost-effective registration of the archaeological heritage. Journal of Archaeological Science 40(2), pp.1108-1121.

Dietrich, J. T. 2015. Riverscape mapping with helicopter-based Structure-from-Motion photogrammetry. Geomorphology, 252, pp. $144-157$.

Forlani, G., Roncella, R., Diotri, F. 2013. Production of highresolution digital terrain models in mountain regions to support risk assessment. Geomatics, Natural Hazards and Risk, Vol. 6, pp. 379397, doi:10.1080/19475705.2013.862746.

Glira, P., Pfeifer, N., Briese, C., Ressl, C., 2015. A correspondence framework for ALS strip adjustments based on variants of the ICP algorithm. PFG Photogrammetrie, Fernerkundung, Geoinformation, 4, pp. 275-289, doi:10.1127/pfg/2015/0270.

James, M. R. Robson, S., 2012. Straightforward reconstruction of 3D surfaces and topography with a camera: accuracy and geoscience application. Journal of Geophysical Research - Earth Surface, 117, p. F03017, doi:10.1029/2011JF002289.

James, M. R. and Robson, S., 2014. Mitigating systematic error in topographic models derived from UAV and ground-based image networks. Earth Surf. Proc. Land. 39, pp. 1413-1420, doi:10.1002/esp.3609.

Karel, W., Doneus, M., Verhoeven, G., Briese, C., Ressl, C., Pfeifer, N., 2013. OrientAL - automatic geo-referencing and orthorectification of archeological aerial photographs. In: ISPRS Annals of the Photogrammetry, Remote Sensing and Spatial Information Sciences, II-5/W1. poster presentation: International Symposium of CIPA, Strasbourg, France; 2013-09-02 - 2013-09-06.

Micheletti, N., Chandler, J. H., Lane, S. N., 2014. Investigating the geomorphological potential of freely available and accessible Structure-from-Motion photogrammetry using a smartphone. Earth Surf. Proc. Land., 40, pp. 473-486, doi:10.1002/esp.3648.

Nocerino, E., Menna, F., Remondino, F., Saleri, R., 2013. Accuracy and block deformation analysis in automatic UAV and Terrestrial photogrammetry-Lesson learnt. ISPRS Annals of the Photogrammetry, Remote Sensing and Spatial Information Sciences, Vol. II-5/W1, XXIV International CIPA Symposium, 2-6 September, Strasbourg, France.

Nocerino, E., Menna, F., Remondino, F., 2014. Accuracy of typical photogrammetric networks in cultural heritage 3D modeling projects. ISPRS-International Archives of the Photogrammetry, Remote Sensing and Spatial Information Sciences, 1, pp. 465-472.

Piermattei, L., Carturan, L., and Guarnieri, A., 2015. Use of terrestrial photogrammetry based on structure from motion for mass balance estimation of a small glacier in the Italian Alps. Earth Surf. Proc. Land., 40, pp. 1791-1802, doi:10.1002/esp.3756, 2015.

Remondino F, Fraser C. 2006. Digital camera calibration methods: considerations and comparisons. Int. Arch. Photogramm. Remote Sens. Spatial Inf. Sci, 36(5), pp. 266-272.

Rothermel, M., Wenzel, K., Fritsch, D., Haala, N., 2012. SURE: photogrammetric surface reconstruction from imagery. Proceedings LC3D Workshop, Berlin, Germany.

Rumpler, M., Daftry, S., Tscharf, A., Prettenthaler, R., Hoppe, C., Mayer, G., Bischof, H. 2014. Automated end-to-end workflow for precise and geo-accurate reconstructions using fiducial markers. ISPRS Ann. Photogramm. Remote Sens. Spatial Inf. Sci., Vol. II-3, pp. 135-142, doi:10.5194/isprsannals-II-3-135-2014.

Smith, M. W., and Vericat, D. 2015. From experimental plots to experimental landscapes: topography, erosion and deposition in subhumid badlands from Structure-from-Motion photogrammetry. Earth Surf. Proc. Land., 40, pp. 1656-1671, doi: 10.1002/esp.3747.

Stumpf, A., Malet, J. P., Allemand, P., Pierrot-Deseilligny, M., Skupinski, G. 2015. Ground-based multi-view photogrammetry for the monitoring of landslide deformation and erosion. Geomorphology, 231, pp. 130-145.

Thoeni, K., Giacomini, A., Murtagh, R., Kniest, E. 2014. A comparison of multi-view 3D reconstruction of a rock wall using several cameras and a laser scanner. Int. Arch. Photogramm. Remote Sens. Spat. Inf. Sci, ISPRS Technical Commission V Symposium, 23-25 June, Riva del Garda, Italy, pp. 573-580.

Wackrow R, Chandler J. 2011. Minimising systematic error surfaces in digital elevation models using oblique convergent imagery. The Photogrammetric Record, 26(133), pp.16-31.

Wenzel, K., Rothermel, M., Fritsch, D. Haala, N., 2013. Image acquisition and model selection for multi-view stereo. Int. Arch. Photogramm. Remote Sens. Spatial Inf. Sci, pp. 251-258. 